\title{
Umedecimento do substrato na germinação de sementes de seis espécies ornamentais ${ }^{(1)}$
}

\author{
KELLI PIROLA(2), MARCELO DOTTO(2), DARCIELI APARECIDA CASSOL ${ }^{(2)}$, \\ AMÉRICO WAGNER JUNIOR(3), PAULO CÉSAR CONCEIÇÃO(3), SÉRGIO MIGUEL MAZARO(3)
}

\begin{abstract}
RESUMO
O objetivo deste trabalho foi avaliar a influência de seis níveis de umedecimento do substrato sobre a germinação das espécies ornamentais Centaurea sp., Petunia sp., Convolvulus sp., Dhalia sp., Adonis sp. e Chrysanthemum sp.. Foram realizados dois experimentos no Laboratório de Fisiologia Vegetal, da Universidade Tecnológica Federal do Paraná - Campus Dois Vizinhos. No experimento 1, o delineamento experimental adotado foi em blocos casualizados, em fatorial 6 x 6 (espécie ornamental x volume de água), com quatro repetições de 50 sementes. No segundo experimento o delineamento diferiu pela ausência do uso de sementes de Dhalia sp. O primeiro e segundo experimento tiveram seus substratos umedecidos, uniformemente, com adição de água destilada, em seis diferentes quantidades, sendo estes de $0,100,150,200,250,300 \mathrm{~mL}$ por $100 \mathrm{~g}$ de Vermiculita ${ }^{\circledR}$ no experimento 1 e $400 \mathrm{~g}$ de Mecplant $(\AA$ no experimento 2. Aos 14 e 21 dias da semeadura, nos experimentos 1 e 2 foram avaliados a porcentagem de germinação e o índice de velocidade de emergência, respectivamente. Com o uso do substrato Vermiculita ${ }^{\circledR}$, recomenda-se para as espécies Dhalia sp. e Chrysanthemum sp. todos os volumes de água testados; para Petunia sp. acima de $150 \mathrm{~mL}$; para Convolvulus sp. os volumes de 200 e $250 \mathrm{~mL}$ de água; para Centeaurea sp., de 150 e $200 \mathrm{~mL}$ de água. Com o uso do substrato Mecplant ${ }^{\mathbb{R}}$, recomenda-se para Chrysanthemum sp., Convolvulus sp., Centeaurea sp. e Petunia sp., todos os volumes de água utilizados. Para Adonis sp., independente do substrato utilizado, novos estudos serão necessários, já que as sementes praticamente não germinaram, podendo este fato estar relacionado com algum tipo de dormência presente na mesma. Palavras-chave: propagação sexuada, vigor, plântulas.
\end{abstract}

\begin{abstract}
Moistening of the substrate on seed germination of six ornamental species

The aim of this study was to evaluate the influence of six wetting levels of the substrate on the germination of Centaurea sp., Petunia sp., Convolvulus sp., Dhalia sp., Adonis sp. and Chrysanthemum sp. ornamental species. Two experiments were carried out in the Laboratory of Plant Physiology, Universidade Tecnológica Federal do Paraná - Campus Dois Vizinhos. In the first experiment, the experimental design was in randomized blocks, in a factorial 6 x 6 (ornamental species x water volume), with four replications of 50 seeds. In the second experiment, the delineation differed by the absence of the use of Dhalia sp. Seeds. The first and second experiment had their substrates moistened uniformly with addition of distilled water at six different quantities, $0,100,150,200,250$ and $300 \mathrm{~mL}$ per $100 \mathrm{~g}$ of Vermiculite ${ }^{\circledR}$ on the experiment 1 and $400 \mathrm{~g}$ of Mecplant ${ }^{\circledR}$ in experiment 2. The germination percentage and emergence rate index were evaluated after 14 and 21 days of sowing, in experiments 1 and 2, respectively. With the use of Vermiculite ${ }^{\circledR}$ it was recommended for Dhalia sp. and Chrysanthemum sp. all the water volumes tested; for Petunia sp. above $150 \mathrm{~mL}$, for Convolvulus sp. 200 and $250 \mathrm{~mL}$ of water and for Centeaurea sp., 150 and $200 \mathrm{~mL}$ of water. The use of the substrate Mecplant ${ }^{\circledR}$ it was recommended for Chrysanthemum sp., Convolvulus sp., Centeaurea sp. and Petunia sp. In all of the volumes of water tested. For Adonis sp., regardless of the substrate used, other studies will be necessary, since that didn't occurred germination, what can be related to some dormancy. Keywords: sexual propagation, vigor, plantlet.
\end{abstract}

\section{INTRODUÇÃO}

O setor da floricultura no Brasil vem apresentando rápido crescimento, tornando-se alternativa viável como atividade agrícola, pois demanda pouca área e geralmente o ciclo de produção é curto, o que permite giro rápido de capital (SILVEIRA e MINAMI, 1997).
A comercialização de sementes de diversas espécies ornamentais, geralmente é destinada para a produção de mudas que serão utilizadas em projetos paisagísticos, ornamentação de vasos, cultivos etc.

Para que as sementes possam ser comercializadas é importante que as mesmas apresentem qualidade fisiológica que permita germinação rápida e uniforme, seguido pela

\footnotetext{
(1) Trabalho recebido para publicação em 12/07/2012 e aprovado em 26/02/2015

(2) Universidade Tecnológica Federal do Paraná (UFPR)- Campus Pato Branco. Via do Conhecimento, Km 01, CEP 85503-390, Pato Branco - PR.

*Autor correspondente: kelli pirola1@hotmail.com.

(3) Universidade Tecnológica Federal do Paraná (UFPR) - Campus Dois Vizinhos. Estrada para Boa Esperança, Km 04, CEP 85660-000, Dois

Vizinhos-PR
} 
emergência de plântulas vigorosas (BRASIL, 2009). Além disso, quando se trata de espécies ornamentais é necessário conhecer as condições adequadas para germinação das sementes de determinada espécie, principalmente pelas respostas diferenciadas que podem apresentar devido a diversos fatores como dormência, condições ambientais, agentes patogênicos entre outros (CARVALHO e NAKAGAWA, 2000).

A germinação é uma sequência ordenada de atividades metabólicas (BEWLEY e BLACK, 1994), sendo a disponibilidade de água um dos principais fatores desencadeantes do processo (VARELA et al., 2005). A hidratação das sementes maturas, secas e não dormentes estabelece o início do processo de germinação, possibilitando a reativação do sistema metabólico e a síntese de novos compostos (CARVALHO e NAKAGAWA, 2000).

Durante esse processo, a absorção de água promove o amolecimento do tegumento, o aumento do volume do embrião e dos tecidos de reserva, favorecendo a ruptura do tegumento, a difusão gasosa e a emergência da raiz primária. Além disso, proporciona a diluição do protoplasma, permitindo a difusão de hormônios e, consequentemente, a ativação de sistemas enzimáticos e, com isso, desenvolvese a digestão, translocação e a assimilação das reservas, resultando no crescimento do embrião (MARCOS FILHO, 2005).

Apesar das sementes necessitarem de certo grau de umidade para iniciar o processo germinativo, Wagner Júnior et al. (2006) descreveram que quantidades excessivas de água podem reduzir a permeabilidade do tegumento das sementes ao oxigênio, levando à inibição da germinação.

Porém, a quantidade inicial de água a ser adicionada no teste de germinação depende da natureza do substrato e, principalmente, das exigências de cada espécie, devendo ser previamente determinada para que sempre seja usada a mesma quantidade nos testes de rotina (FIGLIOLIA et al., 1993).

Assim, o conhecimento das condições adequadas relacionadas à quantidade de água necessária para a germinação de sementes de determinada espécie é de fundamental importância, principalmente pelas respostas diferenciadas que podem ocorrer quando relacionada às exigências de cada uma (NASSIF et al., 1998).

Os estudos de germinação relacionados ao teor de umidade do substrato, contribuem para auxiliar na condução de testes de germinação e redução da discrepância entre os resultados obtidos em laboratório.

Pesquisas sobre as exigências de água em sementes de algumas espécies realizadas por Novembre e Marcos Filho (1999); Varela et al. (2005) e Martins et al. (2009), têm mostrado resultados consistentes, obtidos quando o teor de umidade é controlado através do cálculo baseado na relação volume de água e peso do substrato sem hidratação posterior.

Neste sentido, tornam-se necessários estudos envolvendo as exigências hídricas para germinação de sementes de plantas ornamentais, principalmente pelo aumento da procura por estas, sendo as informações ainda escassas, porém, necessárias.
Sendo assim, o objetivo deste trabalho foi avaliar o efeito de seis níveis de umedecimento do substrato sobre o processo germinativo de seis espécies ornamentais.

\section{MATERIAL E MÉTODOS}

Foram realizados dois experimentos no Laboratório de Fisiologia Vegetal, da Universidade Tecnológica Federal do Paraná - Campus Dois Vizinhos.

Testaram-se sementes comerciais de seis espécies ornamentais (Centaurea sp., Petunia sp., Convolvulus sp., Dhalia sp., Adonis sp. e Chrysanthemum sp.), sendo que a espécie Dhalia sp somente testada no experimento 1.

As sementes testadas nos dois experimentos foram retiradas das embalagens, nas quais foram comercializadas e semeadas na profundidade de $0,5 \mathrm{~cm}$, em caixas Tetra Pak (10 cm x 15,5 cm x 6,5 cm), utilizando como substrato Vermiculita ${ }^{\circledR}$ (mineral com a estrutura da mica que é expandida em fornos de alta temperatura formado essencialmente por silicatos hidratados de alumínio e magnésio) no experimento 1 e Mecplant ${ }^{\circledR}$ (produzido a partir da casca de Pinus bio-estabilizada) no experimento 2.

Em ambos os experimentos, os substratos foram umedecidos, uniformemente, com adição de água destilada, em seis diferentes níveis $(0 ; 100 ; 150 ; 200 ; 250 ; 300 \mathrm{~mL}$ $100 \mathrm{~g}^{-1}$ de Vermiculita ${ }^{\circledR}$ ou $400 \mathrm{~g}^{-1}$ de Mecplant $\left.{ }^{\circledR}\right)$, o que correspondem a $0 ; 1,0 ; 1,5 ; 2,0 ; 2,5 ; 3,0$ e 0,$25 ; 0,375 ; 0,5$; 0,$625 ; 0,75$ vezes o peso da Vermiculita ${ }^{\circledR}$ e do Mecplant ${ }^{\circledR}$ em água, respectivamente.

Antes da adição de água, a Vermiculita ${ }^{\circledR}$ e o Mecplant ${ }^{\circledR}$ foram mantidos a $120^{\circ} \mathrm{C}$ e $60^{\circ} \mathrm{C}$, respectivamente, por 12 horas, para esterilização e secagem, possibilitando obter substrato sem umidade, objetivando maior confiabilidade nos tratamentos testados. Não houve reumedecimento dos substratos durante o decorrer do experimento.

Posteriormente à semeadura, as caixas Tetra Pak $^{\circledR}$ foram envolvidas em saco plástico transparente perfurado, umedecidas e colocadas em câmara de germinação BOD, com temperatura constante de $20^{\circ} \mathrm{C}$, sem iluminação.

No experimento 1, o delineamento experimental adotado foi em blocos casualizados, em fatorial $6 \times 6$ (espécie ornamental $\mathrm{x}$ volume de água), com quatro repetições, considerando-se como unidade experimental, cada lote de 50 sementes.

No experimento 2, o delineamento experimental adotado foi em blocos casualizados, em fatorial $5 \times 6$ (espécie ornamental $\mathrm{x}$ volume de água), com quatro repetições, considerando-se como unidade experimental, cada lote de 50 sementes.

Aos 14 e 21 dias da semeadura (experimentos 1 e 2 , respectivamente), foram avaliados a porcentagem de germinação e o índice de velocidade de emergência (IVE) segundo procedimentos descritos por Maguire (1962). Antes da semeadura foram mensurados o diâmetro e comprimento de cada semente, utilizando paquímetro digital e régua.

Os dados foram submetidos à análise de variância e, para aqueles que se mostraram significativos, foi realizada a comparação de médias pelo teste de Tukey ( $p$ $£ 0,05)$ para fator qualitativo e análise de regressão para 
fator quantitativo, por meio do programa computacional SANEST (ZONTA e MACHADO, 1984).

Os dados das porcentagens de germinação foram transformados previamente antes das análises estatísticas em arco seno $\sqrt{x / 100}$. O IVE foi estabelecido com o teste de emergência e suas avaliações foram realizadas diariamente a partir do surgimento das primeiras plântulas normais (terceiro dia após a semeadura até o décimo quarto dia ou até o vigésimo primeiro dia (experimentos 1 e 2 , respectivamente).

\section{RESULTADOS E DISCUSSÃO}

No experimento 1, os dados de germinação e IVE foram estatisticamente significativos para a interação espécie ornamental x volume de água, bem como, para cada fator isolado (Tabelas 1 e 2 , respectivamente).

De acordo com a Tabela 1, verificou-se que Dhalia sp. e Chrysanthemum sp. tiveram bons resultados de germinação com umedecimento do substrato independente do volume utilizado. Para Petunia sp. houve a necessidade de um volume de água igual ou acima de $150 \mathrm{~mL}$ para obtenção das maiores germinações (Tabela 1). Entretanto, para Convolvulus sp. e Centaurea sp., as maiores médias de germinação foram obtidas quando utilizaram-se volumes de 200 e $250 \mathrm{~mL}$ e 150 e $200 \mathrm{~mL}$, respectivamente, diminuindo-a com os volumes de $300 \mathrm{~mL}$ para Convulvulus sp. e de 250 e $300 \mathrm{~mL}$ para Centaurea sp., podendo este ter sido prejudicado pela falta de oxigênio.

Tabela 1. Germinação (\%) de sementes de seis espécies ornamentais (Centaurea sp., Petunia sp., Convolvulus sp., Dhalia sp., Adonis sp. e Chrysanthemum sp.), de acordo com o volume de água utilizado para umedecer o substratoVermiculita ${ }^{\circledR}$. Table 1. Germination(\%) of six ornamental species (Centaurea sp., Petunia sp., Convolvulus sp., Dhalia sp., Adonis sp. and Chrysanthemum sp.), according the water volume used for it to moisten the Vermiculita ${ }^{\circledR}$ substrate.

\begin{tabular}{|c|c|c|c|c|c|c|c|}
\hline \multirow{2}{*}{ Espécie } & \multicolumn{7}{|c|}{ Volume de água (mL $100 \mathrm{~g}^{-1}$ de vermiculita $\left.{ }^{\circledR}\right)$} \\
\hline & $\mathbf{0}$ & 100 & 150 & 200 & 250 & 300 & Média \\
\hline Dhalia sp. & $0,38 \mathrm{aB}^{*}$ & $93,83 \mathrm{aA}$ & $86,12 \mathrm{aA}$ & $90,05 \mathrm{aA}$ & $92,28 \mathrm{aA}$ & $95.00 \mathrm{aA}$ & 77,44 \\
\hline Adonis sp. & $0,73 \mathrm{aA}$ & 00,0 eA & $00,25 \mathrm{eA}$ & $00,25 \mathrm{dA}$ & $00,25 \mathrm{eA}$ & $00,0 \mathrm{dA}$ & 00,15 \\
\hline Chrysanthemum sp. & $1,46 \mathrm{aB}$ & $72,71 \mathrm{bA}$ & $78,08 \mathrm{abA}$ & $77,64 \mathrm{abA}$ & $72,61 \mathrm{abA}$ & $75,87 \mathrm{bA}$ & 62,19 \\
\hline Convolvulus sp. & $0,00 \mathrm{aC}$ & $15,82 \mathrm{~dB}$ & $23,66 \mathrm{~dB}$ & $50,0 \mathrm{cA}$ & $52,51 \mathrm{cA}$ & $27,46 \mathrm{cB}$ & 23,90 \\
\hline Centaurea sp. & $0,00 \mathrm{aD}$ & $28,91 \mathrm{cdBC}$ & $47,47 \mathrm{cAB}$ & $59,07 \mathrm{bcA}$ & $30,44 \mathrm{dBC}$ & $17,93 \mathrm{cC}$ & 26,23 \\
\hline Petunia sp. & $0,00 \mathrm{aC}$ & $40,71 \mathrm{cB}$ & $63,9 \mathrm{bcA}$ & $72,94 \mathrm{abA}$ & $67,67 \mathrm{bcA}$ & $67,81 \mathrm{bA}$ & 47,71 \\
\hline Média & 0,20 & 38,40 & 46,74 & 55,85 & 51,30 & 44,12 & \\
\hline CV $(\%)$ & & & & 24,60 & & & \\
\hline
\end{tabular}

"Médias seguidas com letras diferentes, minúscula na mesma coluna e maiúscula na mesma linha, diferem significativamente ao nível de $5 \%$ de probabilidade pelo teste de Tukey.

Tanaka et al. (1991) avaliando a influência da quantidade de água no substrato sobre a germinação de sementes de amendoim, verificaram que o maior volume de água utilizado (3 vezes o peso do substrato), foi o que proporcionou a menor germinação, o que possivelmente tenha sido provocada pela deficiência no suprimento de oxigênio, que é um dos fatores essenciais no processo germinativo.

Quanto ao IVE (Tabela 2), verificou-se que volumes iguais ou acima de $100 \mathrm{~mL}$ de água proporcionaram a maior uniformidade de germinação com as espécies Dhalia sp. e Chrysanthemum sp. Resultados semelhantes foram conseguidos com a Petunia sp., diferindo-se apenas pela necessidade de volume igual ou maior a $150 \mathrm{~mL}$ de água. Já para Convolvulus sp e Centaurea sp., a maior uniformidade de germinação ocorreu com os volumes de 200 e $250 \mathrm{~mL}$ e $200 \mathrm{~mL}$, respectivamente.

Tabela 2. Índice de velocidade de emergência de seis espécies ornamentais (Centaurea sp., Petunia sp.,Convolvulus sp.,Dhalia sp., Adonis sp. e Chrysanthemum sp.) de acordo com o volume de água utilizado para umedecer o substrato Vermiculita ${ }^{\circledR}$.

Table 2. Index speed emergence of six ornamental species (Centaurea sp., Petunia sp., Convolvulus sp., Dhalia sp., Adonis sp. and Chrysanthemum sp.), according the water volume used for it to moisten the Vermiculita ${ }^{\circledR}$ substrate.

\begin{tabular}{|c|c|c|c|c|c|c|c|}
\hline \multirow{2}{*}{ Espécie } & \multicolumn{7}{|c|}{ Volume de água (mL $100 \mathrm{~g}^{-1}$ de vermiculitaÒ) } \\
\hline & 0 & 100 & 150 & 200 & 250 & 300 & Média \\
\hline Dhalia sp. & $0,05 \mathrm{aB} *$ & $11,54 \mathrm{aA}$ & $11,36 \mathrm{aA}$ & $12,10 \mathrm{abA}$ & $13,21 \mathrm{aA}$ & $13,31 \mathrm{aA}$ & 9,30 \\
\hline Adonis sp. & $0,05 \mathrm{aA}$ & $00,00 \mathrm{dA}$ & $00,03 \mathrm{cA}$ & $00,03 \mathrm{cA}$ & $00,03 \mathrm{cA}$ & $00,0 \mathrm{dA}$ & 0,02 \\
\hline Chrysanthemum sp. & $0,07 \mathrm{aB}$ & $08,72 \mathrm{aA}$ & $10,17 \mathrm{aA}$ & $10,52 \mathrm{abA}$ & $12,00 \mathrm{aA}$ & $11,54 \mathrm{aA}$ & 8,03 \\
\hline Convolvulus sp. & $0,00 \mathrm{aD}$ & $01,90 \mathrm{cC}$ & $04,06 \mathrm{bB}$ & $09,65 \mathrm{bA}$ & $11,28 \mathrm{aA}$ & $04,55 \mathrm{bB}$ & 4,50 \\
\hline Centaurea sp. & $0,00 \mathrm{aE}$ & $04,82 \mathrm{bC}$ & $09,46 \mathrm{aB}$ & $13,11 \mathrm{abA}$ & $04,78 \mathrm{bC}$ & $02,20 \mathrm{cD}$ & 4,92 \\
\hline Petunia sp. & $0,00 \mathrm{aC}$ & $08,97 \mathrm{aB}$ & $12,63 \mathrm{aA}$ & $14,00 \mathrm{aA}$ & $13,72 \mathrm{aA}$ & $14,32 \mathrm{aA}$ & 9,53 \\
\hline Média & 0,02 & 05,20 & 07,07 & 08,94 & 08,14 & 06,45 & \\
\hline CV $(\%)$ & & & & & & & \\
\hline
\end{tabular}

*Médias seguidas com letras diferentes, minúscula na mesma coluna e maiúscula na mesma linha, diferem significativamente ao nível de 5\% de probabilidade pelo teste de Tukey. 
Pelos resultados obtidos para espécie Adonis sp. quanto à germinação e IVE observou-se que as sementes mesmo quando fornecida maior quantidade de água não apresentaram respostas germinativa e de vigor satisfatórias, o que pode estar relacionado à presença de algum fenômeno de dormência (Tabelas 1 e 2).

Comparando-se as espécies dentro de cada volume de água verificou-se que com $0 \mathrm{~mL}$ não houve diferenças entre as espécies em ambas variáveis (Tabelas 1 e 2), o que já era esperado. Entretanto, com 100, 150, 200, 250 e $300 \mathrm{~mL}$ a maior percentagem de germinação foi obtida com Dhalia sp., que igualou-se ao volume de $200 \mathrm{~mL}$ com Petunia sp. e de $250 \mathrm{~mL}$ com o Chrysanthemum sp.

Supõe-se que esta superioridade obtida com os maiores volumes de água para Dhalia sp. (250 e $300 \mathrm{~mL})$ e Chrysanthemum sp. $(250 \mathrm{~mL})$ possa estar relacionada ao maior tamanho que suas sementes apresentam [Dhalia sp. $\left(18 \mathrm{~mm}^{2}\right)$ e Chrysanthemum sp. $\left.\left(10 \mathrm{~mm}^{2}\right)\right]$, em comparação as demais [Petunia sp. $\left(2 \mathrm{~mm}^{2}\right)$, Centeaura sp. $\left(6 \mathrm{~mm}^{2}\right)$ e Convolvulus sp. $\left.\left(6 \mathrm{~mm}^{2}\right)\right]$, permitindo absorver maior quantidade de água sem que ocorresse prejuízos ao processo germinativo, além de que estas também possuem mais substâncias de reserva.

Segundo Carvalho e Nakagawa (2000), de um modo geral, as sementes maiores podem armazenar mais substâncias de reserva durante a fase de desenvolvimento, o que acarretará em embriões mais desenvolvidos, sendo estas consideradas sementes de maior vigor.
Quanto à Adonis sp, mesmo apresentando o segundo maior tamanho de semente $\left(12 \mathrm{~mm}^{2}\right)$, não apresentou germinação, fato que pode estar ligado à presença de dormência, conforme já ressaltado.

Quanto aos dados de germinação do experimento 2, os mesmos também foram estatisticamente significativos para a interação espécie ornamental $\mathrm{x}$ volume de água, bem como, para cada fator isolado (Tabela 3). Entretanto, no IVE, os resultados foram estatisticamente significativos somente para os fatores isolados (Tabela 4 e Figura 1).

Observou-se que o não umedecimento do substrato, não apresentou germinação para as sementes de Adonis sp. e Chrysanthemum sp.. Para Convulvulus sp., Centeaura sp. e Petunia sp. houve germinação estatisticamente semelhantes entre si, porém baixa, o que pode estar relacionado ao ambiente em que as sementes estavam, podendo ter havido diferença de potencial hídrico entre o meio e as sementes, o que favoreceu o ganho de certa umidade para semente, suficiente para ativar a rota metabólica germinativa de algumas sementes.

Com o umedecimento do substrato, os volumes utilizados dentro de cada espécie, não apresentaram diferenças estatisticamente significativas (Tabela 3).

Quanto ao IVE, em quase todas as espécies, com exceção apenas para Adonis sp., não houve diferença significativa entre as médias obtidas, proporcionando boa uniformidade de germinação (Tabela 4).

Tabela 3. Germinação (\%) de sementes de cinco espécies ornamentais (Centaurea sp., Petunia sp., Convolvulus sp., Adonis sp. e Chrysanthemum sp.), de acordo com o volume de água utilizado para umedecer o substrato Mecplant ${ }^{\circledR}$.

Table 3. Germination (\%) of five ornamental species (Centaurea sp., Petunia sp., Convolvulus sp., Dhalia sp., Adonis sp. And Chrysanthemum sp.), according the water volume used for it to moisten the Mecplant ${ }^{\circledR}$ substrate.

\begin{tabular}{|c|c|c|c|c|c|c|c|}
\hline \multirow{2}{*}{ Espécie } & \multicolumn{7}{|c|}{ Volume de água ( $\mathrm{mL} 400 \mathrm{~g}^{-1}$ de substrato comercial Mecplant $\left.{ }^{\circledR}\right)$} \\
\hline & $\mathbf{0}$ & 100 & 150 & 200 & 250 & 300 & Média \\
\hline Adonis sp. & $0,00 \mathrm{~b} \mathrm{~A}$ & $0,50 \mathrm{~b} \mathrm{~A}$ & $0,00 \mathrm{~b} \mathrm{~A}$ & $0,00 \mathrm{~b} \mathrm{~A}$ & $0,00 \mathrm{~b} \mathrm{~A}$ & $0,00 \mathrm{~b} \mathrm{~A}$ & 00,01 \\
\hline Chrysanthemum sp. & $0,00 \mathrm{bB}$ & 44,36 a A & 67,42 a $A$ & 68,83 a $A$ & $68,41 \mathrm{aA}$ & $48,21 \mathrm{aA}$ & 45,00 \\
\hline Convolvulus sp. & 17,53 a B & 55,48 a $\mathrm{AB}$ & 68,77 a $\mathrm{A}$ & 70,89 a $\mathrm{A}$ & 32,77 a $\mathrm{AB}$ & 44,30 a $\mathrm{AB}$ & 47,94 \\
\hline Centaurea sp. & $4,20 \mathrm{ab} B$ & 43,15 a A & 70,31 a $A$ & 69,57 a A & 63,56 a A & 64,08 a A & 50,71 \\
\hline Petunia sp. & $3,80 \mathrm{ab} B$ & 44,42 a $A$ & 38,20 a $A$ & 76,07 a A & 63,61 a $A$ & 69,13 a $A$ & 47,41 \\
\hline Média & 2,76 & 33,50 & 43,52 & 51,97 & 40,11 & 39,65 & \\
\hline CV (\%) & \multicolumn{6}{|l|}{44,00} & \\
\hline
\end{tabular}

${ }^{*}$ Médias seguidas com letras diferentes, minúscula na mesma coluna e maiúscula na mesma linha, diferem significativamente ao nível de $5 \%$ de probabilidade pelo teste de Tukey,

Tabela 4. Índice de velocidade de emergência de cinco espécies ornamentais (Centaurea sp., Petunia sp.,Convolvulus sp., Adonis sp. e Chrysanthemum sp.).

Table 4. Index speed emergence of five ornamental species (Centaurea sp., Petunia sp., Convulvolus sp., Dhalia sp., Adonis sp. and Chrysanthemum sp.).

\begin{tabular}{l|l} 
Espécie & IVE \\
\hline Adonis sp. & $00,01 \mathrm{~b}^{*}$ \\
\hline Chrysanthemum sp. & $04,88 \mathrm{a}$ \\
\hline Convolvulus sp. & $05,30 \mathrm{a}$ \\
\hline Centaureasp. & $04,05 \mathrm{a}$ \\
\hline Petuniasp & $05,26 \mathrm{a}$ \\
\hline CV (\%) & 28,97
\end{tabular}

*Médias com letras diferentes, minúscula na mesma coluna diferem significativamente ao nível de $5 \%$ de probabilidade pelo teste de Duncan. 


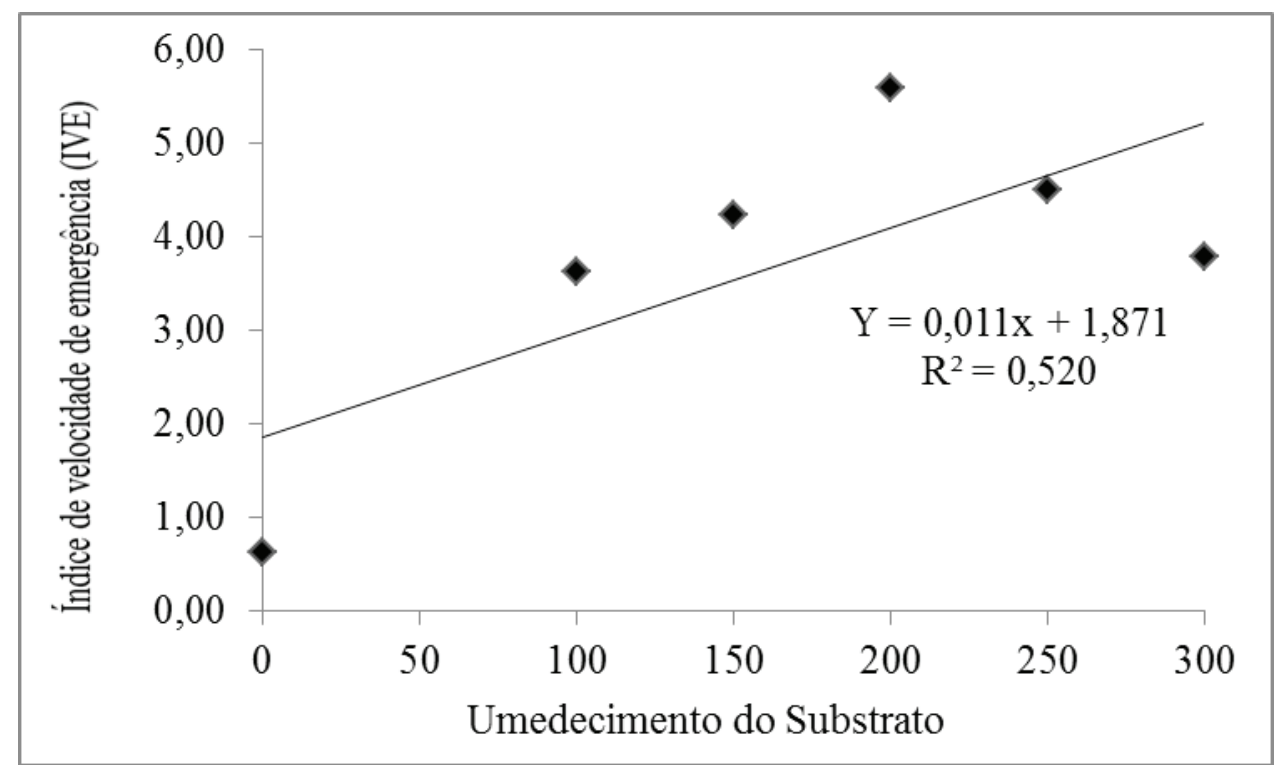

Figura 1. Índice de velocidade de emergência de acordo com o volume de água utilizado para umedecer o substrato Mecplant ${ }^{\circledR}$.

Figure 1. Index speed emergence of according the water volume used for it to moisten the Mecplant ${ }^{\circledR}$ substrate.

Já, ao verificar o volume de água necessário para máximo vigor das sementes, obteve-se ponto de máximo com o volume de $170 \mathrm{~mL}$, cujo IVE foi 3,74 plântulas dia $^{-1}$ (Figura 1).

Em geral, ao analisar ambos os experimentos, observouse que o uso de Vermiculita ${ }^{\circledR}$ em comparação ao Mecplant ${ }^{\circledR}$ (Tabelas 1 e 3) possibilitou aumento na percentagem de sementes germinadas para Chrysanthemum sp. Por outro lado, a Vermiculita ${ }^{\circledR}$ apesar de apresentar como uma das principais características físicas a maior capacidade de retenção de água em relação ao Mecplant ${ }^{\circledR}$ não possibilitou germinação com médias maiores para duas espécies (Centaurea sp. e Convolvulus sp.). O Mecplant ${ }^{\circledR}$ também $^{-}$ favoreceu para que ocorresse germinação mesmo sem o umedecimento do substrato.

Isso pode estar relacionado com a diferença de potencial hídrico ocorrido, conforme ressaltado anteriormente, ou à temperatura utilizada para secagem prévia de ambos os substratos, uma vez que para Mecplant ${ }^{\circledR}$ utilizou-se temperatura de $60{ }^{\circ} \mathrm{C}$, o que não retira a água denominada como higroscópica que fica retida em tensões de Yw entre -3,1 e $-1.000 \mathrm{MPa}$, o que a torna removida da partícula do mesmo em temperaturas superiores a $100^{\circ} \mathrm{C}$, fato este trabalhado com Vermiculita $^{\circledR}$. Com isso, acredita-se que o substrato Mecplant ${ }^{\circledR}$ apresentava antes do uso dos teores de água, porcentagem de umidade maior que de Vermiculita ${ }^{\circledR}$, favorecendo para maior germinação de Centaurea sp. e Convolvulus sp., além de possibilitar também a germinação com $0 \mathrm{~mL}$ de água.

\section{CONCLUSÕES}

Com o uso do substrato Vermiculita ${ }^{\circledR}$, recomenda-se para as espécies Dhalia sp. e Chrysanthemum sp. todos os volumes de água utilizados; para Petunia sp. volumes de água acima de $150 \mathrm{~mL}$; para Convolvulus sp. volumes de 200 e $250 \mathrm{~mL}$ de água e para Centeaurea sp. os volumes de 150 e $200 \mathrm{~mL}$ de água.

Com o uso do substrato Mecplant ${ }^{\circledR}$, recomenda-se para Chrysanthemum sp., Convolvulus sp., Centeaurea sp. e Petunia sp., todos os volumes de água utilizados.

Para Adonis sp., independente do substrato utilizado, novos estudos são necessários, já que as sementes praticamente não germinaram, podendo este fato estar relacionado com algum tipo de dormência.

Ambos os substratos demonstraram potencial em testes germinativos em laboratório.

\section{REFERÊNCIAS}

BEWLEY, J.D.; BLACK, M. Seeds: physiology of development and germination, 2ed. New York: Plenum Press, 1994. 445p.

BRASIL. Ministério da Agricultura, Pecuária e Abastecimento. Regras para análise de sementes. Ministério da Agricultura, Pecuária e Abastecimento. Secretaria de Defesa Agropecuária. Brasília, DF: Mapa/ ACS, 2009. 399 p.

CARVALHO, N.M.; NAKAGAWA, J. Sementes: ciência, tecnologia e produção, 4ed. Jaboticabal: FUNEP, 2000, 588 p.

FIGLIOLIA,M.B.;OLIVEIRA,E.C.;PIÑA-RODRIGUES, F.C.M. Análise de sementes, In: AGUIAR, J.B.; PIÑARODRIGUES, F.C.M.; FIGLIOLIA, M.B. (ed.). Sementes Florestais Tropicais. Brasília: Abrates, p. 134-174, 1993. 
MAGUIRE, J.D. Speed of germination aid in selection and evaluation for emergence and vigour. Crop Science, Madison, v.2, n.2, p.176-177, 1962,

MARCOS FILHO, J. Fisiologia de sementes de plantas cultivadas. Piracicaba: Fealq, 2005. 495p.

MARTINS, C.C.; BOVI, M.L.A.; SPIERING, S.H. Umedecimento do substrato na emergência e vigor de plântulas de pupunheira. Revista Brasileira de Fruticultura. v.31, n.1, p.224-230, 2009.

NASSIF, S.M.N.; VIEIRA, I.G.; FERNADES, G.D. Fatores externos (ambientais) que influenciam na germinação de sementes. Informativo Sementes-IPEF, 1998.

NOVEMBRE, A.D.L.C.; MARCOS FILHO, J. Estudo da metodologia para condução do teste de germinação em sementes de algodão deslintadas mecanicamente. Revista Brasileira de Sementes, Londrina, v.21, n.2, p.187-193, 1999.

SILVEIRA, R.B.A; MINAMI, K. Avaliação da qualidade de crisântemos (Dendranthema grandiflora Tzvelev.), grupo macarrão, produzidos em diferentes regiões do
Estado de São Paulo. Revista Brasileira de Horticultura Ornamental, Campinas, v.3, n.2, p.55-73, 1997.

TANAKA, M.A.S.; MARIANO, M.I.A.; LEÃO, N.V.M. Influência da quantidade de água no substrato sobre a germinação de sementes de amendoim. Revista Brasileira de Sementes, Londrina, v.13, n.1, p.73-76, 1991.

VARELA, V.P.; RAMOS, M.B.P.; MELO, M.F.F. Umedecimento do substrato e temperatura na germinação de sementes de angelim-pedra (Dinizia excelsa Ducke). Revista Brasileira de Sementes, Londrina, v.27, n.2, p.130-135, 2005.

WAGNER JÚNIOR, A.; ALEXANDRE, R.S.; NEGREIROS, J.R.S.; PARIZZOTTO, A.; BRUCKNER, C.H. Influência da escarificação e do tempo de embebição das sementes sobre a germinação de maracujazeiro (Passiflora edulis f. flavicarpa Degener). Revista Ceres, Viçosa, v.52, n.301, p.369-378, 2006.

ZONTA, E.; MACHADO, A.A. SANEST - Sistema de análise estatística para microcomputadores, Pelotas: UFPel, 1984. 75 p. 